\title{
THE WEAKLY COMPACT APPROXIMATION OF THE PROJECTIVE TENSOR PRODUCT OF BANACH SPACES
}

\author{
Qingying Bu \\ University of Mississippi, Department of Mathematics \\ University, MS 38677, U.S.A.; qbu@olemiss.edu
}

\begin{abstract}
We show that $X \hat{\otimes} Y$, the projective tensor product of Banach spaces $X$ and $Y$, has the (bounded) compact approximation property if and only if both $X$ and $Y$ have the same property. We also show that $X \hat{\otimes} Y$ has the weakly compact approximation property (W.A.P.) if both $X$ and $Y$ has the W.A.P. and either (i) every bounded linear operator from $X$ (resp. from $Y$ ) to $Y^{*}$ (resp. to $X^{*}$ ) is completely continuous, or (ii) one of $X$ and $Y$ has the Dunford-Pettis property. As a consequence, we show that if $K$ is scattered and $Y$ has the W.A.P., then $C(K)^{*} \hat{\otimes} Y$ has the W.A.P.
\end{abstract}

A Banach space $X$ is said to have the weakly compact approximation property (W.A.P. for short) if there is a finite constant $C \geq 1$ so that for every weakly compact subset $D$ of $X$ and every $\varepsilon>0$ there is a weakly compact operator $T: X \rightarrow X$ satisfying $\|T\| \leq C$ and $\|T(x)-x\|<\varepsilon$ for all $x \in D$. This weakly compact approximation property was introduced by Astala and Tylli in [1] and systematically studied by Odell and Tylli in [7] from the perspective of Banach space theory. Some applications of this property can be found in [1, 10]. All reflexive Banach spaces clearly have the W.A.P. There are few examples of non-reflexive Banach spaces with (or without) the W.A.P. For instance, the classical Banach space $\ell^{1}$ has the W.A.P. while the classical Banach spaces $c_{0}, C[0,1], L^{1}[0,1]$ and the Hardy space $H^{1}$ fail to have the W.A.P. (see $[1,9]$ ). The quasi-reflexive James' space $J$ and its dual $J^{*}$ have the W.A.P. while the related James' tree space JT fails to have the W.A.P. (see [7]). Saksman and Tylli [9] showed that the projective tensor product $\ell^{p} \hat{\otimes} \ell^{q}$ has the W.A.P. whenever $1<p, q<\infty$ (note that $\ell^{p} \hat{\otimes} \ell^{q}$ is not reflexive whenever $1<p \leq q^{\prime}$, here $\left.1 / q+1 / q^{\prime}=1\right)$. In this paper we show that the projective tensor product $X \hat{\otimes} Y$ of Banach spaces $X$ and $Y$ inherits the W.A.P. from both $X$ and $Y$ if either (i) every bounded linear operator from $X$ (resp. from $Y$ ) to $Y^{*}$ (resp. to $X^{*}$ ) is completely continuous, or (ii) one of $X$ and $Y$ has the Dunford-Pettis property. As a consequence, we give new examples of non-reflexive Banach spaces with the W.A.P.

Diestel, Fourie and Swart in [4] mentioned that the (bounded) approximation property is inherited by the projective tensor product $X \hat{\otimes} Y$ from both $X$ and $Y$. In this paper we show that the (bounded) compact approximation property is also inherited by $X \hat{\otimes} Y$ from both $X$ and $Y$.

For a Banach space $X$, let $X^{*}$ denote its dual space and $B_{X}$ denote its closed unit ball. For vector spaces $X$ and $Y$, let $X \otimes Y$ denote the algebraic tensor product of $X$ and $Y$, and for $A \subseteq X$ and $B \subseteq Y$, let $A \otimes B$ denote the set $\{x \otimes y: x \in A, y \in B\}$.

doi:10.5186/aasfm.2012.3737

2010 Mathematics Subject Classification: Primary 46B28, 46B42.

Key words: (Weakly) compact approximation property, projective tensor product. 
Moreover, if $X$ and $Y$ are Banach spaces, let $X \hat{\otimes} Y$ denote the projective tensor product of $X$ and $Y$, that is, the completion of $X \otimes Y$ in the projective tensor norm

$$
\|u\|_{\wedge}=\inf \left\{\sum_{i=1}^{n}\left\|x_{i}\right\| \cdot\left\|y_{i}\right\|: u=\sum_{i=1}^{n} x_{i} \otimes y_{i}\right\}, \quad u \in X \otimes Y,
$$

where the infimum is taken over all possible representations of $u$. Grothendieck [6] showed that an element $u \in X \hat{\otimes} Y$ if and only if, for every $\varepsilon>0$, there are $x_{n} \in X$ and $y_{n} \in Y$ with $\sum_{n=1}^{\infty}\left\|x_{n}\right\| \cdot\left\|y_{n}\right\|<\infty$ such that

$$
u=\sum_{n=1}^{\infty} x_{n} \otimes y_{n}(\text { converges absolutely in } X \hat{\otimes} Y)
$$

and

$$
\|u\|_{\wedge} \leq \sum_{n=1}^{\infty}\left\|x_{n}\right\| \cdot\left\|y_{n}\right\| \leq\|u\|_{\wedge}+\varepsilon .
$$

From this representation, it is easy to see that

$$
B_{X \hat{\otimes} Y}=\overline{c o}\left(B_{X} \otimes B_{Y}\right) .
$$

For Banach spaces $X_{1}, X_{2}, Y_{1}, Y_{2}$, and bounded linear operators $T_{1}: X_{1} \rightarrow Y_{1}$ and $T_{2}: X_{2} \rightarrow Y_{2}$, one can define a bounded linear operator $T_{1} \otimes T_{2}:\left(X_{1} \otimes X_{2},\|\cdot\|_{\wedge}\right) \rightarrow$ $\left(Y_{1} \otimes Y_{2},\|\cdot\|_{\wedge}\right)$ by

$$
\left(T_{1} \otimes T_{2}\right)\left(x_{1} \otimes x_{2}\right)=T_{1}\left(x_{1}\right) \otimes T_{2}\left(x_{2}\right), \quad x_{1} \in X_{1}, x_{2} \in X_{2} .
$$

Then $\left\|T_{1} \otimes T_{2}\right\| \leq\left\|T_{1}\right\| \cdot\left\|T_{2}\right\|$. Thus $T_{1} \otimes T_{2}$ can be extended from $X_{1} \hat{\otimes} X_{2}$ to $Y_{1} \hat{\otimes} Y_{2}$, still denoted by $T_{1} \otimes T_{2}$.

Recall that for Banach spaces $X$ and $Y$, a bounded linear operator $T: X \rightarrow Y$ is called completely continuous if $T$ maps weakly convergent sequences in $X$ to norm convergent sequences in $Y$. A Banach space $X$ is said to have the Dunford-Pettis property if, for every Banach space $Y$, every weakly compact operator from $X$ to $Y$ is completely continuous.

In the projective tensor product $X \hat{\otimes} Y$, if $A$ and $B$ are, respectively, weakly compact subsets of $X$ and $Y$ then $A \otimes B$ may not be a weakly compact subset of $X \hat{\otimes} Y$. Indeed, if both $X$ and $Y$ are reflexive then $B_{X}$ and $B_{Y}$ are weakly compact. Since $B_{X \hat{\otimes} Y}=\overline{c o}\left(B_{X} \otimes B_{Y}\right), B_{X} \otimes B_{Y}$ is weakly compact in $X \hat{\otimes} Y$ only in the case that $X \hat{\otimes} Y$ is reflexive, which requires that every bounded linear operator from $X$ to $Y^{*}$ is compact. Diestel and Puglisi in [5, Proposition 2.5] showed that if either $X$ or $Y$ has the Dunford-Pettis property then $A \otimes B$ is a weakly compact subset of $X \hat{\otimes} Y$ whenever $A$ and $B$ are, respectively, weakly compact subsets of $X$ and $Y$. Using the same idea we have the following.

Proposition 1. Let $X$ and $Y$ be Banach spaces such that every bounded linear operator $T: X \rightarrow Y^{*}$ (respectively, $T: Y \rightarrow X^{*}$ ) is completely continuous. If $A$ and $B$ are, respectively, weakly compact subsets of $X$ and $Y$ then $A \otimes B$ is a weakly compact subset of $X \hat{\otimes} Y$.

Proof. By the Eberleiň-Smuliàn theorem, it suffices to show that $A \otimes B$ is weakly sequentially compact. Take a sequence $\left(x_{n} \otimes y_{n}\right)_{n}$ in $A \otimes B$. Then there are a subsequence $\left(x_{n_{k}}\right)_{k}$ of $\left(x_{n}\right)_{n}$ and a subsequence $\left(y_{n_{k}}\right)_{k}$ of $\left(y_{n}\right)_{n}$ such that

$$
\text { weak- } \lim _{k} x_{n_{k}}=x_{0} \in A \text { and weak- } \lim _{k} y_{n_{k}}=y_{0} \in B \text {. }
$$


Next we show that $x_{n_{k}} \otimes y_{n_{k}} \rightarrow x_{0} \otimes y_{0}$ weakly in $X \hat{\otimes} Y$.

Take any $\phi \in(X \hat{\otimes} Y)^{*}$. Since $(X \hat{\otimes} Y)^{*}=L\left(X, Y^{*}\right)$, the space of all bounded linear operators from $X$ to $Y^{*}$, let $T \in L\left(X, Y^{*}\right)$ be corresponding to $\phi$. That is,

$$
\langle x \otimes y, \phi\rangle=\langle T(x), y\rangle, \quad \forall x \in X, \forall y \in Y .
$$

Thus

$$
\begin{aligned}
\mid\left\langle x_{n_{k}} \otimes y_{n_{k}}-x_{0} \otimes y_{0}, \phi\right\rangle & =\left|\left\langle T\left(x_{n_{k}}\right), y_{n_{k}}\right\rangle-\left\langle T\left(x_{0}\right), y_{0}\right\rangle\right| \\
& \leq\left|\left\langle T\left(x_{n_{k}}\right)-T\left(x_{0}\right), y_{n_{k}}\right\rangle\right|+\left|\left\langle T\left(x_{0}\right), y_{n_{k}}-y_{0}\right\rangle\right| \\
& \leq M \cdot\left\|T\left(x_{n_{k}}\right)-T\left(x_{0}\right)\right\|+\left|\left\langle T\left(x_{0}\right), y_{n_{k}}-y_{0}\right\rangle\right|
\end{aligned}
$$

where $M=\sup \{\|y\|: y \in B\}<\infty$. By hypothesis, $T$ is completely continuous and hence, $T\left(x_{n_{k}}\right) \rightarrow T\left(x_{0}\right)$ norm in $Y^{*}$. Note that $y_{n_{k}} \rightarrow y_{0}$ weakly in $Y$. Therefore, $x_{n_{k}} \otimes y_{n_{k}} \rightarrow x_{0} \otimes y_{0}$ weakly in $X \hat{\otimes} Y$.

Saksman and Tylli [9] showed that the W.A.P. is inherited by the projective tensor product $\ell^{p} \hat{\otimes} \ell^{q}(1<p, q<\infty)$. Next we give a sufficient condition for the inheritance of the W.A.P. by the projective tensor product $X \hat{\otimes} Y$.

Theorem 2. (i) If $X$ and $Y$ are Banach spaces such that every bounded linear operator $T: X \rightarrow Y^{*}$ (respectively, $T: Y \rightarrow X^{*}$ ) is completely continuous, then $X \hat{\otimes} Y$ has the W.A.P. if and only if both $X$ and $Y$ have the W.A.P. (ii) If $X$ and $Y$ are Banach spaces such that either $X$ or $Y$ has the Dunford-Pettis property, then $X \hat{\otimes} Y$ has the W.A.P. if and only if both $X$ and $Y$ have the W.A.P.

Proof. Take any weakly compact subset $D$ of $X \hat{\otimes} Y$ and any $\varepsilon>0$. By [5, Theorem 3.1], there exist weakly compact subsets $A_{i}$ of $X$ and weakly compact subsets $B_{i}$ of $Y$ such that $D \subseteq \bigcup_{i=1}^{n} \overline{c o}\left(A_{i} \otimes B_{i}\right)$. Let $A=\bigcup_{i=1}^{n} A_{i}$ and $B=\bigcup_{i=1}^{n} B_{i}$. Then $A$ and $B$ are, respectively, weakly compact subsets of $X$ and $Y$. Moreover, it is easy to see that $D \subseteq \overline{c o}(A \otimes B)$. Let

$$
M_{1}=\sup \{\|x\|: x \in A\} \quad \text { and } \quad M_{2}=\sup \{\|y\|: y \in B\} .
$$

Since $X$ has the W.A.P., there are $C_{1}>0$ and a weakly compact operator $T_{1}: X \rightarrow X$ such that

$$
\left\|T_{1}\right\| \leq C_{1} \quad \text { and } \quad\left\|T_{1}(x)-x\right\| \leq \frac{\varepsilon}{4 M_{2}}, \quad \forall x \in A .
$$

Since $Y$ has the W.A.P., there are $C_{2}>0$ and a weakly compact operator $T_{2}: Y \rightarrow Y$ such that

$$
\left\|T_{2}\right\| \leq C_{2} \quad \text { and } \quad\left\|T_{2}(y)-y\right\| \leq \frac{\varepsilon}{4 M_{1} C_{1}}, \quad \forall y \in B .
$$

Thus for every $x \otimes y \in A \otimes B$, one has

$$
\begin{aligned}
\left\|\left(T_{1} \otimes T_{2}\right)(x \otimes y)-(x \otimes y)\right\| & =\left\|T_{1}(x) \otimes T_{2}(y)-x \otimes y\right\| \\
& \leq\left\|T_{1}(x) \otimes\left(T_{2}(y)-y\right)\right\|+\left\|\left(T_{1}(x)-x\right) \otimes y\right\| \\
& \leq C_{1} M_{1} \cdot \frac{\varepsilon}{4 M_{1} C_{1}}+M_{2} \cdot \frac{\varepsilon}{4 M_{2}}=\varepsilon / 2 .
\end{aligned}
$$

Now for every $u \in D \subseteq \overline{c o}(A \otimes B)$ there is $v \in c o(A \otimes B)$ such that

$$
\|u-v\| \leq \frac{\varepsilon}{2\left(1+C_{1} C_{2}\right)}
$$


Write $v=\sum_{i=1}^{n} t_{i}\left(x_{i} \otimes y_{i}\right)$ where $x_{i} \otimes y_{i} \in A \otimes B$ and $\sum_{i=1}^{n}\left|t_{i}\right| \leq 1$. Then

$$
\left\|\left(T_{1} \otimes T_{2}\right)(v)-v\right\| \leq \sum_{i=1}^{n}\left|t_{i}\right| \cdot\left\|\left(T_{1} \otimes T_{2}\right)\left(x_{i} \otimes y_{i}\right)-\left(x_{i} \otimes y_{i}\right)\right\| \leq \frac{\varepsilon}{2},
$$

which implies that

$$
\begin{aligned}
\left\|\left(T_{1} \otimes T_{2}\right)(u)-u\right\| & \leq\left\|\left(T_{1} \otimes T_{2}\right)(u-v)\right\|+\left\|\left(T_{1} \otimes T_{2}\right)(v)-v\right\|+\|v-u\| \\
& \leq\left(\left\|T_{1}\right\| \cdot\left\|T_{2}\right\|+1\right) \cdot\|u-v\|+\frac{\varepsilon}{2} \leq \varepsilon
\end{aligned}
$$

Moreover, $\left\|T_{1} \otimes T_{2}\right\| \leq\left\|T_{1}\right\| \cdot\left\|T_{2}\right\| \leq C_{1} \cdot C_{2}$. Next we need only to show that $T_{1} \otimes T_{2}: X \hat{\otimes} Y \rightarrow X \hat{\otimes} Y$ is weakly compact.

Since $B_{X \hat{\otimes} Y}=\overline{c o}\left(B_{X} \otimes B_{Y}\right)$, one has

$$
\begin{aligned}
\left(T_{1} \otimes T_{2}\right)\left[B_{X \hat{\otimes} Y}\right] & =\left(T_{1} \otimes T_{2}\right)\left[\overline{c o}\left(B_{X} \otimes B_{Y}\right)\right] \\
& \subseteq \overline{c o}\left(\left(T_{1} \otimes T_{2}\right)\left[B_{X} \otimes B_{Y}\right]\right) \\
& =\overline{c o}\left(T_{1}\left[B_{X}\right] \otimes T_{2}\left[B_{Y}\right]\right) .
\end{aligned}
$$

Note that $T_{1}\left[B_{X}\right]$ and $T_{2}\left[B_{Y}\right]$ are, respectively, relatively weakly compact subsets of $X$ and $Y$. By Proposition 1 and [5, Proposition 2.5], $T_{1}\left[B_{X}\right] \otimes T_{2}\left[B_{Y}\right]$ is a relatively weakly compact subset of $X \hat{\otimes} Y$. It follows from the Kreǐn-Smuliàn theorem that $\overline{c o}\left(T_{1}\left[B_{X}\right] \otimes T_{2}\left[B_{Y}\right]\right)$ is a weakly compact subset of $X \hat{\otimes} Y$ and hence, $\left(T_{1} \otimes T_{2}\right)\left[B_{X \hat{\otimes} Y}\right]$ is a relatively weakly compact subset of $X \hat{\otimes} Y$. This implies that $T_{1} \otimes T_{2}: X \hat{\otimes} Y \rightarrow$ $X \hat{\otimes} Y$ is weakly compact and thus $X \hat{\otimes} Y$ has the W.A.P.

Remark. By applying the construction in [2] to $\ell^{1}$ one obtains a separable $\mathcal{L}^{\infty}$ space $X$ so that $\ell^{1} \subset X$ isometrically and $X$ has the Schur property (also see [9]). It follows from [1, Corollary 3] that $X$ has the W.A.P. Saksman and Tylli in [9] asked a question whether $X \hat{\otimes} X$ has the W.A.P.? Note that the Schur property implies the Dunford-Pettis property. Thus the previous Theorem 2 gives an affirmative answer to this question.

Every reflexive Banach space clearly has the W.A.P. There are a few examples of non-reflexive Banach spaces with the W.A.P. (see [7, 9]). If $K$ is scattered then $C(K)^{*}$ has the Schur property (see [8], also see [4]) and hence, has the DunfordPettis property. It follows from [1, Corollary 3] that $C(K)^{*}$ also has the W.A.P. Thus we have the following corollary that gives us new examples of non-reflexive Banach spaces with the W.A.P.

Corollary 3. Let $K$ be scattered and $Y$ be a Banach space with the W.A.P. Then $C(K)^{*} \hat{\otimes} Y$ has the W.A.P. In particular, $C(K)^{*} \hat{\otimes} C(K)^{*}$ has the W.A.P.

Recall that a Banach space $X$ is said to have the compact approximation property (see [3, p. 308]) if for every compact subset $K$ of $X$ and every $\varepsilon>0$ there is a compact operator $T: X \rightarrow X$ such that $\|T(x)-x\| \leq \varepsilon$ for all $x \in K$. A Banach space $X$ is said to have the bounded compact approximation property (see [3, p. 308]) if there exists $\lambda \geq 1$ so that for every compact subset $K$ of $X$ and every $\varepsilon>0$ there is a compact operator $T: X \rightarrow X$ satisfying $\|T\| \leq \lambda$ and $\|T(x)-x\| \leq \varepsilon$ for all $x \in K$. It is well known that the (bounded) approximation property implies the (bounded) compact approximation property, but the converse is not true (see [11] or see [3, p. 309]). 
Diestel, Fourie and Swart [4] showed that if Banach spaces $X$ and $Y$ have the approximation property (respectively, the bounded approximation property) then the projective tensor product $X \hat{\otimes} Y$ has the same property. To obtain the compact approximation property and the bounded compact approximation property for the projective tensor product $X \hat{\otimes} Y$, we need the following characterization of relatively norm compact subsets in $X \hat{\otimes} Y$ which is due to Grothendieck [6] (also see [5]).

Proposition 4. A subset $K$ of $X \hat{\otimes} Y$ is relatively norm compact if and only if there are compact subsets $A$ of $X$ and $B$ of $Y$ such that $K \subseteq \overline{c o}(A \otimes B)$.

By the previous proposition and following the last part in the proof of Theorem 2, we have the following corollary.

Corollary 5. Let $X_{1}, X_{2}, Y_{1}, Y_{2}$ be Banach spaces. If $T_{1}: X_{1} \rightarrow Y_{1}$ and $T_{2}: X_{2} \rightarrow$ $Y_{2}$ are compact, then $T_{1} \otimes T_{2}: X_{1} \hat{\otimes} X_{2} \rightarrow Y_{1} \hat{\otimes} Y_{2}$ is compact.

Similar to the proof of Theorem 2 we have the following.

Theorem 6. If $X$ and $Y$ are Banach spaces with the compact approximation property (respectively, the bounded compact approximation property), then $X \hat{\otimes} Y$ has the compact approximation property (respectively, the bounded compact approximation property).

\section{References}

[1] Astala, K., and H.-O. Tylli: Seminorms related to weak compactness and to Tauberian operators. - Math. Proc. Cambridge Philos. Soc. 107, 1990, 367-375.

[2] Bourgain, J., and G. Pisier: A construction of $\mathcal{L}^{\infty}$-spaces and related Banach spaces. - Bol. Soc. Brasil. Mat. 14, 1983, 109-123.

[3] Casazza, P. G.: Approximation properties. - In: Handbook of the geometry of Banach spaces, vol. I, edited by Johnson and Lindenstrauss, North-Holland, Amsterdam, 2001, 271-316.

[4] Diestel, J., J. Fourie, and J. Swart: The projective tensor product I. - Contemp. Math. $321,2003,37-65$.

[5] Diestel, J., and D. Puglisi: A note on weakly compact subsets in the projective tensor product of Banach spaces. - J. Math. Anal. Appl. 350, 2009, 508-513.

[6] Grothendieck, A.: Produits tensoriels topologiques et espaces nucleaires. - Mem. Amer. Math. Soc. 16, 1955.

[7] Odell, E., and H.-O. Tylli: Weakly compact approximation in Banach spaces. - Trans. Amer. Math. Soc. 357, 2005, 1125-1159.

[8] Pelczynski, A., and Z. Semadeni: Spaces of continuous functions, III. Spaces $C(\Omega)$ for $\Omega$ without perfect subsetes. - Studia Math. 18, 1959, 211-222.

[9] Saksman, E., and H.-O. Tylli: New examples of weakly compact approximation in Banach spaces. - Ann. Acad. Sci. Fenn. Math. 33, 2008, 429-438.

[10] Tylli, H.-O.: Duality of the weak essential norm. - Proc. Amer. Math. Soc. 129, 2001, 14371442 .

[11] Willis, G.: The compact approximation property does not imply the approximation property. - Studia Math. 103, 1992, 99-108. 